\title{
FEATURES OF TERRA $\backslash$ MOD11A2DAY IN OPERATIONAL FORECASTOF GRAIN CROPS YIELD IN KAZAKHSTAN WITH AN 8 DAY RENEWAL
}

\author{
Alexey Terekhov \\ Kazakh Research Institute of Ecology and Climate, Ministry of Environment, KAZAKHSTAN \\ E-mail: aterekhov1@yandex.ru
}

Commission VIII, WG VIII/6

\begin{abstract}
:
The Kazakhstan, with export capacity of 6-8 million tons, is one of the largest wheat exporter in the world. About 16 million hectares of unirrigated land is used for monocultural cultivation of cereals (wheat and barley). Most of the cropland is located in the steppe and forest steppe zone. The moisture deficit limits the crop productivity and creates a strong dependency of its state of the moisture conditions during vegetation season. In Kazakhstan, the average grain yield variations are sufficiently large, from 0.9 (2010) to 1.4 ton lha (2007). Given the high volatility of the gross grain harvest and export potential, respectively, methods of early satellite forecast of grain yield with high frequency of the renewal are of the great interest.
\end{abstract}

In Kazakhstan, the variations in the weather growing season determine the yield of grain crops. By significant weather parameters include: the spring soil moisture, humidity and air temperature, rainfall, and several others. Plants respond to the sum of all parameters through the volume of green biomass. The regional cereal state can be estimated from satellite vegetation indices, which are particularly informative in the period of its seasonal peak. Another satellite parameter closely related to humidity conditions may be the land surface temperature (LST).

Product USGS: TERRA \MOD11A2DAY represents the 8-days LST composite was tested in the task of estimating of arable lands temperature in Northern Kazakhstan. The description of the temperature conditions of the growing season based on the temperature calibrated index (TCI), which was introduced by Kogan. TCI provides a weighted assessment of the current LST on a scale of 0-100, where 0 - the lowest, respectively, 100 as a high temperature, recorded during the observation period at a given location at a given time window. The monitoring period included 2004-2010 years. During the beginning of the growing season was taken on April 15, season end on 20 August - ripeness stage of wheat.

It was analyzed the two largest agriculture provinces - Akmola and Kostanay with a total cropland size is about 9 million hectares. The size of cultivated areas in Akmola and Kostanay areas during 2004-2010 has undergone a substantial increase, from 6.7 million hectares in 2004 to 8.9 million hectares in 2010. The temperature regime characterized by a weather component, and not depend on the land use type. Therefore, for the diagnosis of arable land in the period 2004-2010 was used a fixed mask, covering most major crop areas.

Description of the temperature regime of arable land during the growing season (April 15-August 20) has the form of TCI time series which consists of 15 values ( 8 day period of renewal). The time window of each of the 15 assessments began on April 15 and ended up floating-date with progressive increments in 8 days. Accordingly, the last value of TCI, reflecting a weighted temperature throughout the analyzed part of the growing season (April 15-August 20). last value of TCI has a good correlation with the official cereal yield, R2 = 0,98 for Akmola, R2 $=0,90$ for Kostanay oblast. These values are higher than the correlation between seasonal maximum NDVI (TERRA \MOD13Q1, 187-203 days) and yield of grain crops, which accounted for R2 =0,85 Akmola, and R2 $=0,73$ for Kostanay oblast.

Scheme of the operative forecast grain yields was based on estimates of the temperature dynamics of arable land current year in comparison with the conditions of the past ones. The closest temperature regimes of previous years, in linear proportion to the degree of closeness to the current year, created a synthetic year-analogue. Yield, the year-analogue consisted of proportionate share of the yield components of its past. The magnitude of this yield, calculated as 15 times a season, forms the operational forecast with 8-day period of renewal.

Thus, the TCI based on TERRA / MOD11A2DAY on informative in assessing the ceeral condition in Northern Kazakhstan is not inferior TERRA / MOD13Q1 / NDVI. Due to variations in sowing date assessment of culture-based vegetation index at the regional scale is difficult in the first half of the growing season, when there is a significant increase in the values of NDVI. LST is not directly connected with the agriculture cultivation techniques. This allows you to weigh the weather conditions and build the scheme of early operational forecasting crop yields with a high renewal rate when there is a close relationship between the temperature regime of arable land and crops condition. 